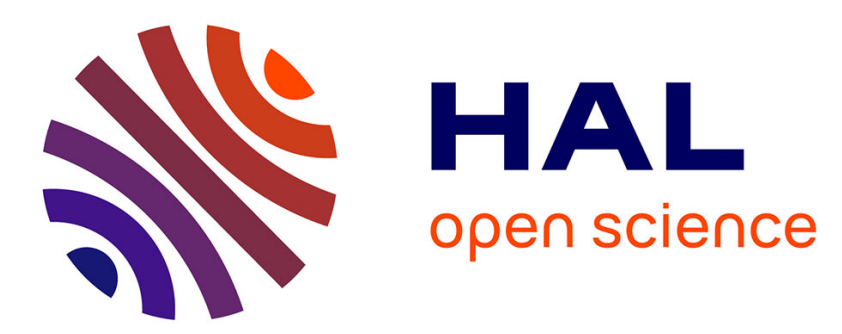

\title{
A differential tube-based model for predicting the linear viscoelastic moduli of polydisperse entangled linear polymers
}

\author{
Adrien Leygue, Christian Bailly, Roland Keunings
}

\section{- To cite this version:}

Adrien Leygue, Christian Bailly, Roland Keunings. A differential tube-based model for predicting the linear viscoelastic moduli of polydisperse entangled linear polymers. Journal of Non-Newtonian Fluid Mechanics, 2006, 133 (1), pp.28-34. 10.1016/j.jnnfm.2005.10.003 . hal-01004701

\section{HAL Id: hal-01004701 \\ https://hal.science/hal-01004701}

Submitted on 21 Jan 2018

HAL is a multi-disciplinary open access archive for the deposit and dissemination of scientific research documents, whether they are published or not. The documents may come from teaching and research institutions in France or abroad, or from public or private research centers.
L'archive ouverte pluridisciplinaire HAL, est destinée au dépôt et à la diffusion de documents scientifiques de niveau recherche, publiés ou non, émanant des établissements d'enseignement et de recherche français ou étrangers, des laboratoires publics ou privés. 


\title{
A differential tube-based model for predicting the linear viscoelastic moduli of polydisperse entangled linear polymers
}

\author{
A. Leygue ${ }^{a}$, C. Bailly ${ }^{b}, *$ R. Keunings ${ }^{a}$ \\ ${ }^{a}$ CESAME, Université catholique de Louvain, B-1348 Louvain-la-Neuve, Belgium \\ ${ }^{\mathrm{b}}$ Unité de Chimie et de Physique des Hauts Polym 'eres, Université catholique de Louvain, B-1348 Louvain-la-Neuve, Belgium
}

\begin{abstract}
We present a simple tube theory for topologically linear entangled polymers that accounts for reptation, contour-length fluctuations and thermal constraint release. This theory is based on a new differential formulation of the thermal constraint release phenomenon proposed by the authors [A.Leygue, C.Bailly, R.Keunings, A differential formulation of thermal constraint release for entangled polymers, J.Non Newtonian Fluid Mech. 128 (1) (2005) 23-28] which is extended here to account for contour-length fluctuations. We apply the theory to mono- and poly-disperse polystyrene melts and demonstrate its ability to produce quantitative predictions. Additionally, we discuss a mathematically linear approximation of our approach that preserves the structure of the model. While most quantitative tube theories for predicting linear viscoelasticity are mathematically non-linear, our approach allows one to address the linear viscoelastic response of a polydisperse entangled system with a mathematically linear theory.
\end{abstract}

Keywords: Entangled linear polymers; Linear visco-elasticity; Polymer mixtures

\section{Introduction}

Tube theories addressing the quantitative prediction of the linear viscoelastic properties of entangled systems based on their microstructure have now reached a high level of maturity. Following the pioneering work of de Gennes [2] on reptation, numerous tube models are now capable of a quantitative description of the linear viscoelastic properties of linear entangled polymers (see e.g. [3-7]). The success of these theories is such that the focus is now on the prediction of the linear viscoelastic response of systems with a much more complex architecture such as mixtures of star and linear polymers [8-10]. For systems involving more complex architectures such as long chain branching, a hierarchical algorithm has been proposed by Larson [11]. Park et al. [12] have modified it to incorporate recent theoretical developments. Despite their focus on the linear rheology of entangled systems, a striking fact about all those theories is that they are mathematically non-linear.

\footnotetext{
* Corresponding author.

E-mail addresses: bailly@poly.ucl.ac.be (C. Bailly), roland.keunings@inma.ucl.ac.be (R. Keunings).
}

In order to predict the relaxation modulus of an entangled polymer, one must describe how the polymeric chains escape from their constraining tubes and how this relaxation process affects the tubes themselves. For linear polymers, the escape of a chain can occur through reptation or contour-length fluctuations [13]. Although they are often addressed separately, these two phenomena can be viewed as different modes of a Rouse chain trapped in a tube potential. For example, the stochastic description of linear chains $[6,14,15]$ does not consider reptation and fluctuations separately as fluctuations correspond to the breathing modes of a one-dimensional Rouse chain escaping from a tube. Different authors have proposed coupled descriptions of reptation and contour-length fluctuations by either considering a position-dependent $[16,17]$ or a time-dependent [18] diffusion process along the primitive path.

Thermal Constraint Release [19] theories address the effects, on the tube, of the relaxation of the surrounding chains. In a sense, constraint release is a closure to the tube potential. Viovy et al. [20] have proposed a rigorous description of constraint release and have shown when Rouse-like tube motion induced by thermal constraint release can be approximated by a dilation of the tube. The double reptation picture $[21,22]$ provides a simple and successful alternative to complex constraint re- 
lease theories. Double reptation actually is a fair approximation of Rouse tube motion. Likthman and McLeish have recently shown that generalized double reptation is essentially correct down to the Rouse time of the chain [6]. For polydisperse systems, double reptation induces a non-linear mixing rule which has actually been recovered in the stochastic reptation model proposed by Hua et al. [14]. A limitation of double reptation appears for polydisperse systems where different chains relax on well separated timescales. In that case, the random pairwise interactions picture of double reptation breaks down, as the fast dynamics of the short chains would induce a very fast relaxation of the slower chains, which is not observed experimentally.

In this paper, we present a simple tube model for mixtures of linear polymers. This theory, valid in the rheologically linear regime only, proposes a coupled description of reptation, contour-length fluctuations and constraint release. Reptation and contour-length fluctuations are accounted for through a position dependent diffusion mechanism proposed by Graham et al. [17], while thermal constraint release is introduced using an extension of a new differential formulation of double reptation [1]. Our theory allows one to compute directly the tube survival probability along the primitive path. Furthermore, the extension to the polydisperse case yields a perfectly linear mixing rule. Then we demonstrate the predictive capabilities of our approach on a large set of polystyrene samples. Finally, we show how it is possible to build a mathematically linear approximation of our theory that retains both the structure of the model and the physical meaning of the variables. The resulting model is therefore mathematically linear (linear equations and mixing rule) and is able to predict quantitatively the linear viscoelastic properties of entangled linear polymers. The next step is of course to build a constitutive equation for the non-linear rheological regime whose linearization would be the linear model presented in this work [23].

\section{Tube theory for a monodisperse system}

In this section we present a tube theory for entangled linear systems. We first consider the monodisperse case and then extend the theory to account for polydispersity.

Let $s(-1 \leq s \leq 1)$ be a parametric coordinate along the primitive path of a polymer chain in a monodisperse environment. Let us define $P_{\gamma}^{f}(t, s)$ the probability for a tube segment with position $s$ to survive between the arbitrary initial time 0 and time $t$. The superscript $f$ denotes that we do account for contour-length fluctuations, while the parameter $\gamma$ is representative of the effectiveness of constraint release. Two limiting cases are $P_{0}^{f}(t, s)$ and $P_{\gamma}(t, s)$. The quantity $P_{0}^{f}(t, s)$ is representative of a chain relaxing in a fixed network of entanglements where no constraint release occurs, while $P_{\gamma}(t, s)$ properly describes a very long chain where the length-fluctuations become negligible. Reptation theory $[2,13]$ suggests that the time evolution of $P_{0}^{f}(t, s)$ is governed by a diffusion operator along the coordinate $s$. This operator represents the diffusive motion of the chain inside its tube that yields the destruction/renewal of a tube segment that reaches the chain ends. Following Des Cloizeaux [16] and Graham [17], we consider that the effect of contour-length fluctuations can be accounted for through a position-dependent diffusion constant. The simplest form for the evolution of $P_{0}^{f}(t, s)$ is therefore [13]:

$\frac{\partial}{\partial t} P_{0}^{f}=\frac{\partial}{\partial s}\left(\alpha_{d}^{f}(s) \frac{\partial}{\partial s} P_{0}^{f}\right)$,

$P_{0}^{f}(t,-1)=0$,

$P_{0}^{f}(t, 1)=0$ for $t>0$,

$P_{0}^{f}(0, s)=1$ for $-1<s<1$,

where Eqs. (2) and (3) come from the assumption that chain ends are fully relaxed.

As suggested in [17] we consider $\alpha_{d}^{f}(s)$ to be constant along the primitive path, except at the chain ends in two regions of size proportional to $\sqrt{M / M_{e}}$, where $M$ is the molecular weight of the polymeric chain and $M_{e}$ is the molecular weight between entanglements. In those regions, where contour-length fluctuations allow for a faster renewal of the tube segments, the diffusion coefficient is proportional to the inverse of the quadratic distance to the chain end. As the molecular weight of the polymer increases, the influence of contour-length fluctuations becomes more and more negligible. In that limiting case, the reptation picture [2] tells us that the diffusion constant should scale like the inverse of the cube of the molecular weight of the polymer. The full expression for $\alpha_{d}^{f}(s)$ becomes:

$$
\begin{aligned}
\alpha_{d}^{f}(s)= & \frac{4}{K_{d} \pi^{2} M^{3}} \frac{K_{f}^{2} M_{e}}{M(1-s)^{2}} \text { if } s>\left(1-K_{f} \sqrt{\frac{M_{e}}{M}}\right), \\
& \frac{4}{K_{d} \pi^{2} M^{3}} \frac{K_{f}^{2} M_{e}}{M(1+s)^{2}} \text { if } s<\left(K_{f} \sqrt{\frac{M_{e}}{M}}-1\right), \\
& \frac{4}{K_{d} \pi^{2} M^{3}} \text { otherwise, }
\end{aligned}
$$

where $K_{d}$ is a material parameter and the $M^{-3}$ dependence of $\alpha_{d}^{f}(s)$ is due to the non-dimensional nature of the spatial parametric coordinate $s$. The adjustable parameter $K_{f}$ is close to unity and controls the depth of the contour-length fluctuations within the model. We do not claim any universal value for $K_{f}$ and consider it as a parameter that has to be identified for each material. This is similar to the parameter $M^{\star}$ of Des Cloizeaux [18] that also controls fluctuations. Taking $K_{f}=0$ actually suppresses the fluctuations as the diffusion constant becomes constant along the chain. In that special case, one recovers the simple reptation picture of Doi and Edwards [13]. The reptation time $\tau_{d}(M)$ can then be defined as:

$\tau_{d}(M)=K_{d} M^{3}$.

At this point, it is worth noticing that the simple scaling we adopt for the width of the fluctuation zone cannot be valid for poorly entangled systems. When $M$ is only a few times $M_{e}$, the reptation picture tends to break down as fluctuations become the dominant relaxation mechanism. 
Finally we account for constraint release using the approach we developed in [1]. This is done by adding a local relaxation term to Eq. (1) representative of relaxation through constraint release. Following the double reptation picture, we postulate that the rate of constraint release is proportional to the rate of relaxation through reptation and contour-length fluctuations, and that constraint release events occur with the same probability on all unrelaxed segments. The first assumption translates the fact that constraint release is actually driven by the other relaxation mechanisms. The second one states that the probability for a segment to relax through constraint release does not depend on its position along the chain. At a given position along the primitive path, the rate of relaxation through constraint release must therefore be proportional to the local tube survival probability. The resulting differential problem for $P_{\gamma}^{f}(t, s)$ reads:

$\frac{\partial P_{\gamma}^{f}}{\partial t}=\frac{\partial}{\partial s}\left(\alpha_{d}^{f}(s) \frac{\partial}{\partial s} P_{\gamma}^{f}\right)+\beta P_{\gamma}^{f}$,

$P_{\gamma}^{f}(t,-1)=0$,

$P_{\gamma}^{f}(t, 1)=0$ for $t>0$,

$P_{\gamma}^{f}(0, s)=1$ for $-1<s<1$,

where $\beta\left(P_{\gamma}^{f}\right)$ is the instantaneous rate of thermal constraint release. We now extend the expression for $\beta\left(P_{\gamma}^{f}\right)$ proposed in [1] to account for both reptation and contour-length fluctuations:

$\beta\left(P_{\gamma}^{f}\right)=\gamma \frac{\int_{-1}^{1} \partial / \partial s\left(\alpha_{d}^{f}(s) \partial / \partial s\left(P_{\gamma}^{f}\right)\right) \mathrm{d} s}{\int_{-1}^{1} P_{\gamma}^{f} \mathrm{~d} s}$,

where $\gamma$ is an adjustable parameter of order unity that controls the amount of constraint release in the system. It is important to notice that $\beta$ is not constant in time and therefore the local relaxation does not obey a simple first-order decay.

The relaxation modulus $G(t)$ is simply defined as being proportional to the average tube survival probability along the chain:

$G(t)=G_{N}^{0} \frac{1}{2} \int_{-1}^{1} P_{\gamma}^{f}(t, s) \mathrm{d} s$,

$G_{N}^{0}$ being the plateau modulus.

In order to predict the high frequency response, the expression (10) for the relaxation modulus has to be modified to account for the Rouse modes of the chain. In practice, this is simply done by superposing a Rouse relaxation modulus $G_{r}(t)$ on the relaxation modulus coming from reptation. Following [5], the expression for the Rouse relaxation modulus for a chain of mass $M$ writes:

$$
\begin{aligned}
G_{r}(t)= & G_{N}^{0}\left(\sum_{p=Z+1}^{\infty} \frac{1}{Z} \exp \left(-\frac{p^{2}}{\tau_{r}}\right)\right. \\
& \left.+\frac{1}{3} \sum_{p=1}^{Z} \frac{1}{Z} \exp \left(-\frac{p^{2}}{\tau_{r}}\right)\right),
\end{aligned}
$$

where $\tau_{r}=K_{r} M^{2}$ and $K_{r}$ is an additional linear material parameter. The integer $Z$ is defined as the closest integer to the ratio $M / M_{e}$. The material parameters $K_{d}, K_{r}$ and $M_{e}$ are not independent as $\tau_{d}(M)$ is linked to $\tau_{r}(M)$ through the underlying segmental dynamics, characterized by the equilibration time $\tau_{e}$. Following Larson et al. [24] we have:

$$
\begin{aligned}
K_{d} & =3 \frac{\tau_{e}}{M_{e}^{3}}, \\
K_{r} & =\frac{\tau_{e}}{M_{e}^{2}} .
\end{aligned}
$$

The independent parameters of the model are therefore $M_{e}, \tau_{e}$, $G_{N}^{0}, K_{f}$ and $\gamma$.

\section{Extension to the polydisperse case}

We now extend the theory presented in the previous section to the polydisperse case, without adding any adjustable parameter. The extension procedure is similar to the one we proposed in [1], where contour-length fluctuations were neglected. Let us consider a polydisperse system of polymers with $N$ different masses $M^{(i)}$. Let $P_{\gamma}^{f(i)}(t, s)$ be the tube survival probability at time $t$ and parametric position $s$ for the chains of mass $M^{(i)}$. We assume that all chains reptate and fluctuate independently of the polydispersity of the system. The rate of constraint release is however the same for all chains and is computed from the relaxation of all masses. The differential problem governing the evolution of $P_{\gamma}^{f(i)}$ reads:

$$
\begin{aligned}
& \frac{\partial P_{\gamma}^{f(i)}}{\partial t}=\frac{\partial}{\partial s}\left(\alpha_{d}^{f(i)}(s) \frac{\partial}{\partial s} P_{\gamma}^{f(i)}\right)+\beta P_{\gamma}^{f(i)}, \\
& P_{\gamma}^{f(i)}(t,-1)=0, \\
& P_{\gamma}^{f(i)}(t, 1)=0 \text { for } t>0, \\
& P_{\gamma}^{f(i)}(0, s)=1 \text { for }-1<s<1 .
\end{aligned}
$$

In view of Eq. (9), the rate of constraint release $\beta$ is consistently defined as:

$\beta=\gamma \frac{\sum_{i} \phi^{(i)} \int_{-1}^{1} \partial / \partial s\left(\alpha_{d}^{f(i)}(s) \partial / \partial s\left(P_{\gamma}^{f(i)}\right)\right) \mathrm{d} s}{\sum_{i} \phi^{(i)} \int_{-1}^{1} P_{\gamma}^{f(i)} \mathrm{d} s}$,

where $\phi^{(i)}$ is the volume fraction of species $(i)$ and $\gamma$ keeps the same meaning as in the monodisperse case. The diffusion coefficient $\alpha_{d}^{f(i)}(s)$ is computed from Eq. (4), where $M^{(i)}$ is substituted for $M$. Similarly to Eq. (10), we define the relaxation modulus as the volume average over the different species of the tube survival probability:

$G(t)=G_{N}^{0} \sum_{i=1}^{N} \frac{\phi^{(i)}}{2} \int_{-1}^{1} P_{\gamma}^{f(i)}(t, s) \mathrm{d} s$.

By directly computing the relevant quantity i.e. the tube survival probability under reptation, contour-length fluctuations and thermal constraint release, we alleviate the need for a non-linear mixing rule.

In order to correctly model the short-time behaviour, the Rouse relaxation modulus (11) can be added for each species using a linear mixing rule. 


\section{Numerical solution of the model}

Although the above theory is quite simple from a mathematical point of view, a closer look at Eq. (14) shows that the differential problems for all masses are coupled through the constraint release term. This is numerically expensive for a large number of masses. The numerical cost can however be reduced dramatically through the use of a semi-analytical solution that decouples the different masses.

In the absence of constraint release, i.e. when $\gamma=0$, the partial differential Eq. (14) become decoupled. The functions $P_{0}^{f(i)}(t, s)$ can therefore be computed numerically at a reasonable cost using a finite difference scheme. Through direct substitution, one can verify that the following expression for $P_{\gamma}^{f(i)}(t, s)$ satisfies Eq. (14):

$P_{\gamma}^{f(i)}(t, s)=P_{0}^{f(i)}(t, s)\left(\sum_{i=1}^{N} \frac{\phi^{(i)}}{2} \int_{-1}^{1} P_{0}^{f(i)}(t, s) \mathrm{d} s\right)^{\gamma}$.

The computation of $P_{\gamma}^{f(i)}$ with this method has a truly negligible numerical cost as, for a large number of masses, the algorithmic complexity is reduced from quadratic to linear in $N$.

\section{Model predictions}

We now present predictions of the model in the monodisperse case. Next, we apply the model for the prediction of linear viscoelastic moduli of different samples of polydisperse polystyrene melts. For all predictions, a Rouse spectrum described by Eq. (11) has been superposed using a linear mixing rule, as done by van Ruymbeke et al. [5].

In Fig. 1, we show the molecular weight dependence of the predicted zero-shear viscosity of a monodisperse system. In the limit of highly entangled chains, the viscosity scales like the cube of the molar mass. As the number of entanglements gets lower, this scaling gradually changes to an exponent 3.4. In this example we chose to set $\gamma=1$ and $K_{f}=0.6$, as these are the values we identified for the polystyrene melts discussed later in this section. The value of the other parameters does not change the shape of the viscosity curve but merely translates it vertically and horizontally. For the sake of completeness, we compare our predictions with those of Likthman and McLeish $\left(C_{v}=1\right)$ [6]. The models predicts similar trends and apparently the same asymptotic value. The differences showing up for less entangled systems are mostly due to differences in the relative importance of contour-length fluctuations between the two models. With $\gamma=1.2$ and $K_{f}=2$ our model predicts a viscosity curve very close to that of Likhtman and McLeish.

To evaluate the model further, we test its ability to correctly predict the linear viscoelastic properties of six polydisperse polystyrene samples. The parameters of the model are estimated using the molecular weight distribution and the viscoelastic moduli of a single sample. The value of the parameters is then frozen for the subsequent predictions on the remaining five samples. The data of the six samples named PS1, PS2, PS3, PS60, PS275 and PS330 were kindly provided to us by BASF.

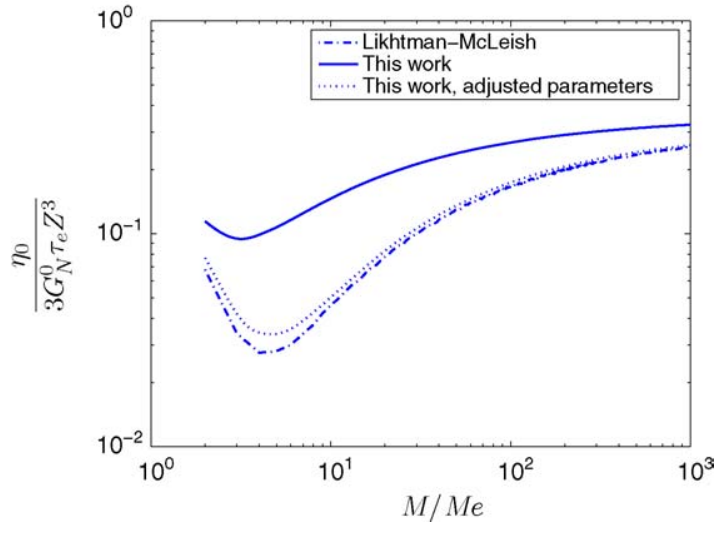

Fig. 1. Dependence of the zero-shear viscosity $\eta_{0}$ with the molar mass for a strictly monodisperse sample. The plain line is the prediction of our model $\left(\gamma=1\right.$ and $K_{f}=0.6$ ), while the dashed line is the prediction of the Likhtman and McLeish model [6]. The dotted line is the prediction of our model where we increased the depth of contour length fluctuations and the effectiveness of constraint release $\left(\gamma=1.2\right.$ and $\left.K_{f}=2\right)$.

We also received the molecular weight distribution and the viscoelastic moduli at $170^{\circ} \mathrm{C}$ from BASF. The characteristics of the various samples are provided in Table 1. Samples PS1, PS60 and PS275 are quite monodisperse, while PS2 and PS330 have a much broader molecular weight distribution. PS3 is a trimodal sample with a fraction of very high masses. The parameter estimation is actually quite a delicate task for two reasons. In the first place, the sensitivity of the model to the different parameters can be very uneven. Second, not all samples are suited to perform this estimation, if they only provide information in a narrow molecular weight range which is not representative of the physics built in the theory. Therefore, we chose to use the broad sample PS3 to fit the parameters of the model. In order to account for the whole molecular weight distribution, we discretize it with a small fixed step in logarithmic space. The efficient semi-analytical method described in Section 4 still allows us to compute quickly the model predictions. The parameters resulting from the fitting procedure are reported in Table 2 . The molecular weight distribution as well as the measured and predicted viscoelastic moduli for PS3 are reported in Fig. 2(a and b). We see there a good quantitative agreement between the data and the predictions, over a wide range of frequencies.

In Figs. 2-4 we show, for a wide range of molecular weight distributions, the quantitative agreement between experimentally measured viscoelastic moduli and the model's predictions. All these predictions were obtained using the single set of parameters from Table 2 . The main discrepancies between predictions and measurements are found at low and high frequencies.

Table 1

Characteristics of the six PS samples provided by BASF

\begin{tabular}{llll}
\hline Name & $M_{w}(\mathrm{~kg} / \mathrm{mol})$ & $M_{n}(\mathrm{~kg} / \mathrm{mol})$ & $M_{w} / M_{n}$ \\
\hline PS1 & 320 & 270 & 1.18 \\
PS2 & 274 & 101 & 2.72 \\
PS3 & 407 & 143 & 2.83 \\
PS60 & 69.6 & 62.7 & 1.11 \\
PS275 & 290 & 253 & 1.07 \\
PS330 & 324 & 112 & 2.89
\end{tabular}



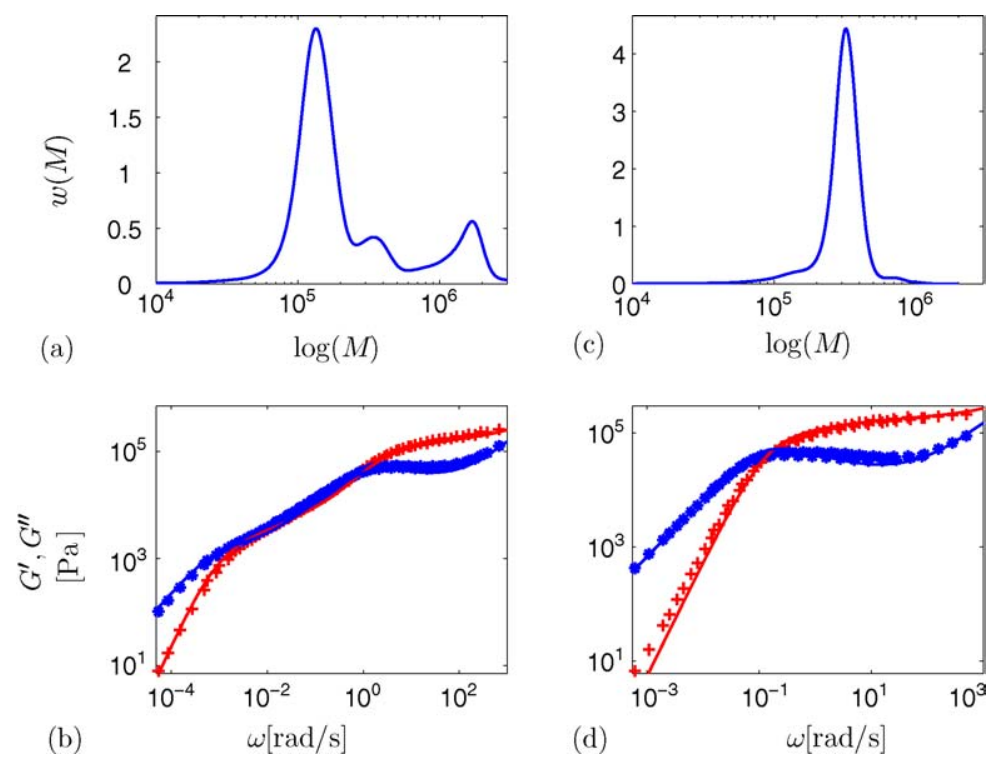

Fig. 2. Molecular weight distribution and linear viscoelastic moduli of PS3 (a and b) and PS1 (c and d). Plain lines are the model's output, while the dots are the experimental data.
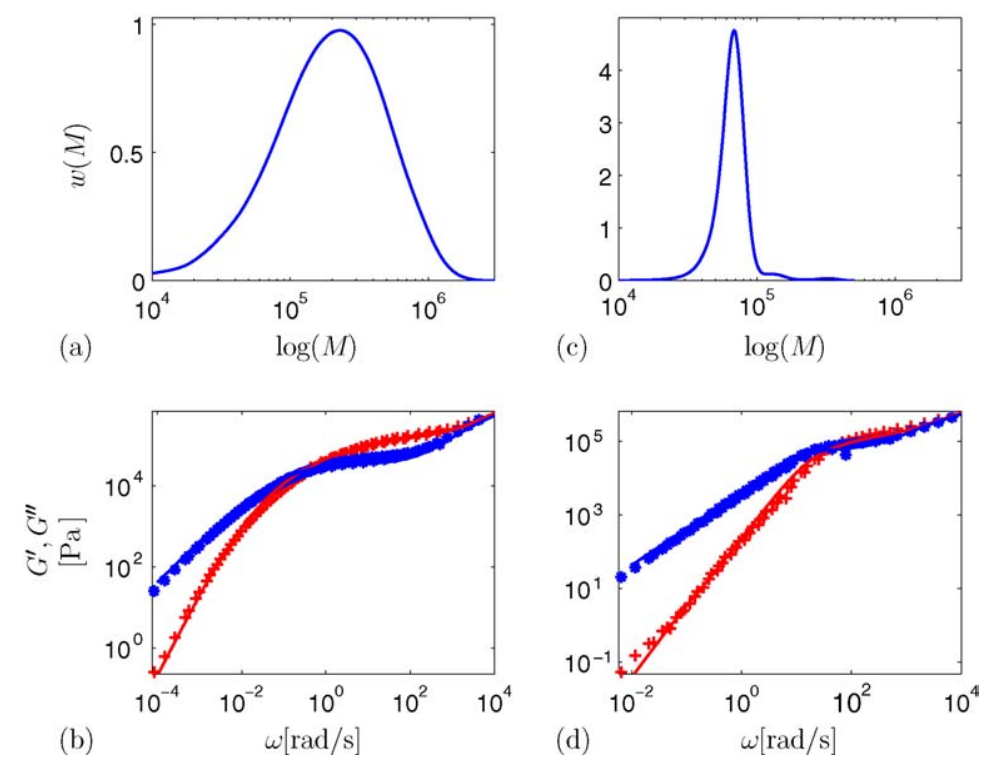

Fig. 3. Molecular weight distribution and linear viscoelastic moduli of PS2 (a and b) and PS60 ( $\mathrm{c}$ and d). Plain lines are the model's output, while the dots are the experimental data.

At low frequency, the model sometimes fails to correctly predict the storage modulus. In this frequency range the dominating mechanisms are reptation and constraint release. Furthermore, the parameter $\gamma$, which controls constraint release, has the main influence on the slope of the storage modulus in this region. We would therefore suggest that a more accurate treatment of constraint release would help resolve these discrepancies.

Table 2

Parameters resulting from the fitting of the model on the PS3 data

\begin{tabular}{ll}
\hline$G_{N}^{0}$ & $2 \times 10^{5} \mathrm{~Pa}$ \\
$\tau_{e}$ & $5.0 \times 10^{-4} \mathrm{~s}$ \\
$M_{e}$ & $15700 \mathrm{~g} / \mathrm{mol}$ \\
$K_{f}$ & 0.6 \\
$\gamma$ & 1.0 \\
\hline
\end{tabular}

At intermediate and high frequencies the loss modulus tends to be underestimated. We connect this feature to the crude description of contour-length fluctuations we implemented.

\section{Linear approximation of the theory}

Despite the relative simplicity of our theory, it remains mathematically non-linear. The non-linear operator on $P_{\gamma}^{f}(t, s)$ found in the definition (9) makes the rate of constraint release $\beta$ time dependent. This non-linearity is not a problem per se but having to rely on a mathematically non-linear theory to describe linear rheological properties of a system prevents us from building a theory for the rheologicaly non-linear regime on top of the previous one. In the present section we propose a simple ap- 

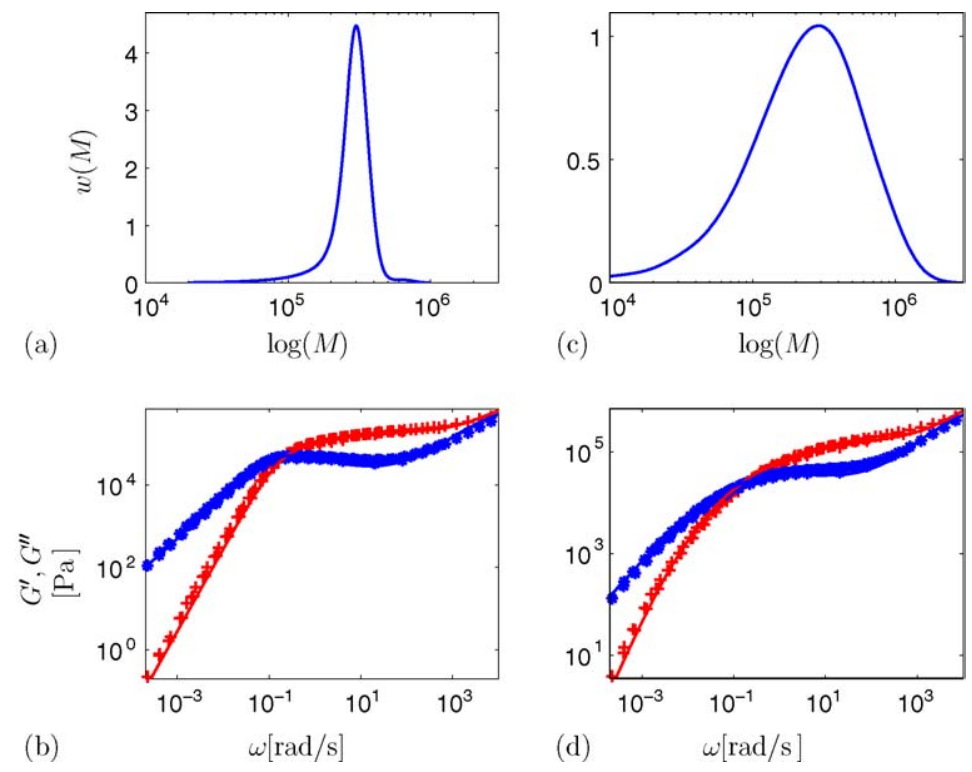

Fig. 4. Molecular weight distribution and linear viscoelastic moduli of PS275 (a and b) and PS330 (c and d). Plain lines are the model's output, while the dots are the experimental data.

proximation of our theory that preserves its general mathematical structure, but where both the mixing rule and the operators acting on the variables of the model are mathematically linear.

The non-linearity of our theory comes from the timedependent relaxation induced by constraint release. Therefore, we propose to approximate constraint release through a superposition of few linear modes. Let us call $Q_{\mathrm{CR}}(t)$ or constraint release kernel the second factor of the right-hand-side of Eq. (17). This factor represents the effects of constraint release on $P_{0}^{f(i)}(t, s)$ :

$Q_{\mathrm{CR}}(t)=\left(\sum_{i=1}^{N} \frac{\phi^{(i)}}{2} \int_{-1}^{1} P_{0}^{f(i)}(t, s) \mathrm{d} s\right)^{\gamma}$.

Let us assume that the constraint release kernel $Q_{\mathrm{CR}}(t)$ can be approximated by a finite sum of decreasing time-exponentials:

$Q_{\mathrm{CR}}(t) \approx \sum_{j=0}^{N_{\mathrm{CR}}} w_{j} \exp \left(-t / \tau_{j}\right)$

where $w_{j}$ and $\tau_{j}$ are adjustable parameters. For a given of $N$, we compute $w_{j}$ and $\tau_{j}$ using a minimisation procedure for the the quadratic error between the exact constraint release kernel (18) and its approximation (19). We can now define $P_{\gamma}^{f(i, j)}(t, s)$ as the solution of the following linear differential problem:

$\frac{\partial P_{\gamma}^{f(i, j)}}{t}=\frac{\partial}{\partial s}\left(\alpha_{d}^{f(i)}(s) \frac{\partial}{\partial s} P_{\gamma}^{f(i, j)}\right)-\frac{1}{\tau_{j}} P_{\gamma}^{f(i, j)}$,

$P_{\gamma}^{f(i, j)}(t,-1)=0$

$P_{\gamma}^{f(i, j)}(t, 1)=0$ for $t>0$,

$P_{\gamma}^{f(i, j)}(0, s)=1$ for $-1<s<1$.
As for Eq. (14), the analytical solution of Eq. (20) can be expressed as a function of $P_{0}^{f(i)}$ :

$P_{\gamma}^{f(i, j)}(t, s)=P_{0}^{f(i)}(t, s) \exp \left(-t / \tau_{j}\right)$.

Making use of Eq. (19), one can verify that the following expression for the relaxation modulus correctly approximates Eq. (16) of the original theory:

$G(t)=G_{N}^{0} \sum_{i=0}^{N}\left(\frac{\phi^{(i)}}{2} \int_{-1}^{1}\left(\sum_{j=1}^{N_{\mathrm{CR}}} P_{\gamma}^{f(i, j)}(t, s)\right) \mathrm{d} s\right)$.

In the previous expression, the sub-expression contained within the innermost parenthesis actually is an approximation of $P_{\gamma}^{f(i)}(t, s)$. The quality of this linear approximation depends only on the quality of the approximation of the constraint release kernel (18) by a sum of decreasing exponentials. In Fig. 5, we show

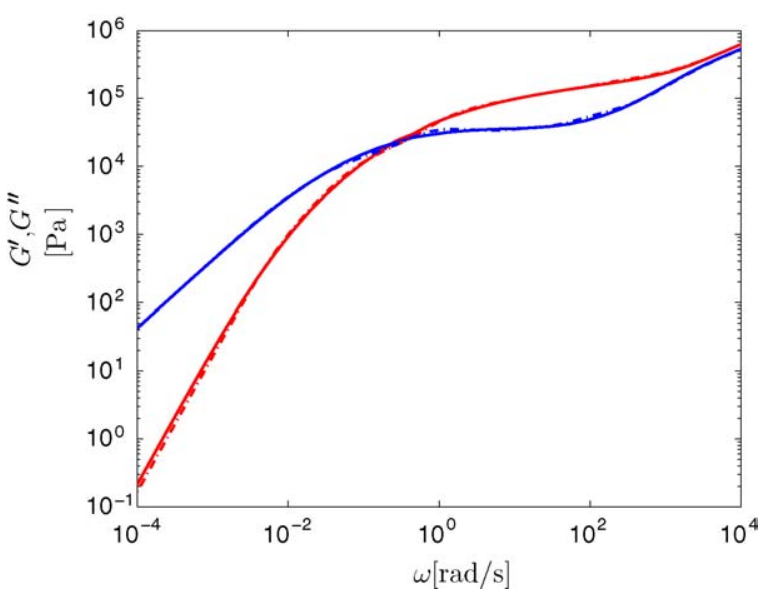

Fig. 5. Comparison between the viscoelastic moduli of the full model (-) and its linear approximation(---.-). Seven modes were used to approximate the constraint release kernel of the full model. 
for sample PS2 that, with seven modes, the linear approximation is almost indistinguishable from the original model.

\section{Conclusions}

We have presented a simple tube theory for linear entangled systems. The specificity of this theory is that, for a monodisperse system, a single partial differential equation is used to compute the tube survival probability under reptation, contourlength fluctuations and thermal constraint release. Reptation and contour-length fluctuations are modelled simultaneously by position-dependent diffusion along the primitive path. Thermal constraint release is introduced through a time dependent relaxation term. The intensity of the thermal constraint release is controlled by reptation and contour-length fluctuations. In a polydisperse environment, we directly compute the tube survival probability under the influence of all masses with a set of coupled partial differential equations. Additionally, we proposed a semi-analytical method for the numerical solution of our model. This method reduces the algorithmic complexity from quadratic to linear in terms of the number of masses, thus making the computational cost negligible even for a broad molecular weight distribution.

Although some discrepancies remain, we have shown the good predictive properties of our theory on a wide range of molten polystyrene samples.

Finally, we have proposed an approximation of our theory, that enables us to address the linear viscoelastic properties of entangled linear polymers with a mathematically linear theory. This approximation is suited as a starting point for building constitutive equations for the non-linear regime. Work on this topic is in progress.

\section{Acknowledgements}

This work is supported by the "Action de recherche concertée" programme of the Communauté Franç aise de Belgique and by "BASF Aktiengesellschaft". The authors wish to thank Dr. Martin Laun for making the PS data available.

\section{References}

[1] A. Leygue, C. Bailly, R. Keunings, A differential formulation of thermal constraint release for entangled polymers, J. Non Newtonian Fluid Mech. 128 (1) (2005) 23-28.

[2] P.G. De, Gennes, Reptation of a polymer chain in the presence of fixed obstacles, J. Chem. Phys. 55 (1971) 572-579.

[3] C. Pattamaprom, R.G. Larson, T.J. Van Dyke, Quantitative predictions of linear viscoelastic rheological properties of entangled polymers, Rheol. Acta 39 (6) (2000) 517-531.
[4] F. Léonardi, J.-C. Majesté, A. Allal, G. Marin, Rheological models based on the double reptation mixing rule: the effects of a polydisperse environment, J. Rheol. 44 (2000) 675-692.

[5] E. van Ruymbeke, R. Keunings, V. Stéphenne, A. Hagenaars, C. Bailly, Evaluation of reptation models for predicting the linear viscoelastic properties of entangled linear polymers, Macromolecules 35 (7) (2002) 26892699.

[6] A.E. Likhtman, T.C.B. McLeish, Quantative theory for linear dynamics of linear entangled polymers, Macromolecules 35 (2002) 6332-6343.

[7] S.T. Milner, T.C.B. McLeish, Reptation and contour-length fluctuations in melts of linear polymers, Phys. Rev. Lett. 81 (3) (1998) 725-728.

[8] S.T. Milner, T.C.B. McLeish, R.N. Young, A. Hakiki, J.M. Johnson, Dynamic dilution, constraint-release, and star-linear blends, Macromolecules 31 (1998) 9345-9353.

[9] J.H. Lee, L.A. Archer, Stress relaxation of star/linear polymer blends, Macromolecules 35 (2002) 6687-6696.

[10] E. van Ruymbeke, R. Keunings, C. Bailly, Prediction of linear viscoelastic properties for polydisperse mixtures of entangled star and linear polymers: modified tube-based model and comparison with experimental results, J. Non Newtonian Fluid Mech. 128 (1) (2005) 7-22.

[11] R.G. Larson, Combinatorial rheology of branched polymer melts, Macromolecules 34 (13) (2001) 4556-4571.

[12] S.J. Park, S. Shanbhag, R.G. Larson, A hierarchical algorithm for predicting the linear viscoelastic properties of polymer melts with long-chain branching, Rheol. Acta 44 (3) (2005) 319-330.

[13] M. Doi, S.F. Edwards, The Theory of Polymer Dynamics, Oxford University Press, 1986.

[14] C.C. Hua, J.D. Schieber, D.C. Venerus, Segment connectivity, chain-length breathing, segmental stretch, and constraint release in reptation models. i. theory and single-step strain predictions, J. Chem. Phys. 109 (22) (1998) 10018-10032.

[15] Y. Masubuchi, J. Takimoto, K. Koyama, G. Ianniruberto, G. Marrucci, F. Grecco, Brownian simulations of a network of reptating primitive chains, J. Chem. Phys. 115 (9) (2001) 4387-4394.

[16] J. Des Cloizeaux, Relaxation of entangled polymers in melts, Macromolecules 23 (17) (1990) 3992-4006.

[17] R.S. Graham, A.E. Likhtman, T.C.B. McLeish, S.T. Milner, Microscopic theory of linear entangled polymer chains under rapid deformation including chain stretch and convective constraint release, J. Rheol. 47 (5) (2003) 1171-1200.

[18] J. Des Cloizeaux, Relaxation and viscosity anomaly of melts made of long entangled polymers, Macromolecules 23 (21) (1990) 4678-4687.

[19] G. Marrucci, Relaxation by reptation and tube enlargement: A model for polydisperse polymers, J. Polym. Sci., Polym. Phys. 23 (1985) 159177.

[20] J.L. Viovy, M. Rubinstein, R.H. Colby, Constraint release in polymer melts: tube reorganization versus tube dilation, Macromolecules 24 (12) (1991) 3587-3596.

[21] C. Tsenoglou, Viscoelasticity of binary polymer blends, ACS Polym. Prepr 28 (1987) 185-186.

[22] J. Des Cloizeaux, Double reptation versus simple reptation in polymer melts, J. Europhys. Lett. 5 (1988) 437-442.

[23] A. Leygue, C. Bailly, R. Keunings. A tube-based constitutive equation for polydisperse entangled linear polymers, J. Non Newtonian Fluid Mech., submitted for publication, 2005.

[24] L.G. Leal, G.H. McKinley, A.E. Likhtman, R.G. Larson, T. Sridhar, T.C.B. McLeish, Definitions of entanglement spacing and time constants in the tube model, J. Rheol. 47 (2003) 809-818. 